\title{
Impacto de la Plataforma MOODLE como soporte tecnológico al desarrollo de cursos presenciales en la FAREM-Carazo, UNAN-Managua durante el primer semestre del año 2017
}

Ing. Wilber José Matus González UNAN-Managua, FAREM-Carazo Departamento de Ciencias, Tecnología y Salud wilberj1987@hotmail.com

DOI: https://doi.org/10.5377/torreon.v6i17.6874

Palabras Clave: Educación, Virtual, Moodle, Soporte, Semipresencial

\section{RESUMEN}

on el objetivo de evaluar el impacto de la Plataforma Moodle como soporte tecnológico al desarrollo de cursos presenciales en la FAREM-Carazo, UNANManagua durante el primer semestre del año 2017, se realizó un estudio descriptivo, correlacional y analítico, en el que fueron analizados los datos sobre el nivel de satisfacción de los estudiantes y docentes con respecto al uso de la plataforma Moodle, además, se analizaron las características que poseen los cursos Moodle implementados en la FAREMCarazo, en los cuales se logró determinar que existe un alto nivel de satisfacción de parte de los estudiantes, con un $75.72 \%$ de tendencia positiva; por otro lado, gracias a la colaboración de los docentes de la FAREM-Carazo que implementaron cursos Moodle durante el primer semestre del 2017, se identificó cuáles eran los beneficios y dificultades de la utilización de Moodle basados en su experiencia, y por medio de un análisis de los diferentes cursos implementados por estos docentes se identificó las características comunes de estos cursos.

\section{INTRODUCCIÓN}

En el mundo se están experimentando cambios importantes en todos los ámbitos del quehacer humano, uno de ellos es el acceso al conocimiento. Vivimos en lo que llamamos la era 
del conocimiento y el desarrollo tecnológico, la que permite hoy acceder a grandes recursos de información, procesarlos y transformarlos para servir de apoyo a la inteligencia y memoria de las personas. La tecnología está cambiando radicalmente las formas de trabajo y los medios a través de los cuales las personas se comunican y aprenden.

La tecnología se ha venido incorporando al ámbito académico, y el desarrollo de la informática en los años sesenta consolida la utilización de ordenadores con fines educativos, concentrándose en la enseñanza asistida por computadoras, asimismo, con la aparición de las computadoras personales, esta opción tomó dimensiones mayores. Para los años ochenta, llegan bajo la denominación de Nuevas Tecnologías de la Información y la Comunicación Renovadas, opciones que están apoyadas en el desarrollo de máquinas y dispositivos diseñados para almacenar, procesar y transmitir de modo flexible, grandes cantidades de información.

Los cursos en línea implementados en la plataforma Moodle son una de las modalidades de la educación a distancia y semipresencial que se utilizan en diversos ámbitos educativos en la actualidad, esta utiliza Internet con todas sus herramientas tecnológicas de la información y la comunicación para realizar el proceso de enseñanza-aprendizaje. Este método de enseñanza se consolida como uno de los avances más importantes que ha tenido la tecnología dentro del ámbito educativo.

Moodle es una plataforma que trae muchos beneficios a los procesos de información, interacción y comunicación, como señala Cabero (2006). Moodle pone a disposición de los alumnos un amplio volumen de información, además de ofrecer diferentes herramientas de comunicación sincrónica y asincrónica para los estudiantes y docentes.

La UNAN-Managua en su proceso de mejora continua, se plantea la utilizaciónde diversas metodologías y técnicas para el apoyo de la enseñanza. Entre estos se encuentra la implementación de la educación en línea, la cual, por medio de la creación de actividades virtuales en un entorno educativo, comprende diferentes momentos tales como la concepción, la planeación, la construcción, aplicación y valoración de las mismas.

La presente investigación busca conocer el impacto de la plataforma Moodle como soporte a las clases presenciales de la UNAN-Managua, FAREM-Carazo, principalmente en el nivel de satisfacción de los estudiantes y docentes, así como los beneficios y dificultades que estos presentan.

\section{Objetivos}

El presente estudio se desarrolla en el marco del trabajo de tesis para optar al título de Máster en Métodos de Investigación Científica, de la UNAN-Managua, y busca conocer el 
impacto de Moodle dentro del ámbito de la Educación Superior de esta facultad, para lo cual se plantearon los siguientes objetivos:

1. Caracterizar el nivel de aceptación respecto a los cursos Moodle por parte de estudiantes de la FAREM-Carazo.

2. Determinar los beneficios y dificultades en el uso de plataforma Moodle de parte de docentes de la FAREM-Carazo.

3. Identificar elementos comunes en los cursos que desarrollan los docentes de la FAREM-Carazo en la Plataforma Moodle.

4. Analizar el nivel de interacción de los estudiantes matriculados en los cursos que están en la plataforma MOODLE de la FAREM-Carazo.

5. Crear un manual técnico-metodológico para el diseño y gestión de cursos Moodle.

\section{METODOLOGÍA}

La herramienta de evaluación utilizada para obtener la información de los alumnos fue un cuestionario diseñado para este propósito, aplicado a una muestra de 346 alumnos perteneciente a las diferentes titulaciones que oferta la FAREM-Carazo. Esta muestra se obtuvo por medio del muestreo aleatorio simple para poblaciones finitas. El cuestionario se presenta con un acápite de datos generales y una escala Likert y su propósito fue medir el nivel de satisfacción de los estudiantes respecto al uso de la plataforma. Este instrumento consiste en un conjunto de ítems bajo la forma de afirmaciones o juicios, ante los cuales se solicitó la reacción (favorable o desfavorable, positiva o negativa) de los individuos. Los alumnos dispusieron de cinco opciones de respuesta, por cada afirmación planteada, entre las que se incluyen: totalmente insatisfecho, insatisfecho, no sabe o no opina, satisfecho y totalmente satisfecho.

Así mismo, se hizo uso de un muestreo no probabilístico para la selección de la muestra de docentes para la aplicación del instrumento respectivo. Se tomó en consideración a los docentes de esta facultad que desarrollaron cursos Moodle en el primer semestre del 2017 y mantuvieron los cursos activos a lo largo del periodo en estudio. El tamaño de muestra no probabilístico de acuerdo al criterio basado en expertos se determinó usando el procedimiento definido por Arias (2012) quien define que el propio investigador es capaz de seleccionar a los sujetos que considere apropiado.

Por otro lado, se realizó un grupo focal con el fin de conocer la experiencia de los docentes en la utilización de cursos Moodle. Este fue dirigido a todos aquellos docentes que hubiesen tenido más de dos cursos durante el semestre. 
Este trabajo se realizó bajo el enfoque de investigación Mixta, con equilibrio de peso entre el enfoque cuantitativo y el cualitativo. Son muchos los autores que en la actualidad recomiendan el uso de ambos enfoques, a fin de complementar los beneficios de cada uno de ellos, orientándose así hacia la triangulación metodológica, destacando en este caso, el planteamiento de análisis de datos cuantitativos e información cualitativa propuesto por Hernández, Fernández y Baptista (2014).

De acuerdo a la naturaleza de cada una de las variables y guiados por el compromiso definido en cada uno de los objetivos específicos, se realizaron los análisis descriptivos correspondientes. En la parte cuantitativa se hizo el análisis de frecuencia y estadísticas descriptivas según cada caso. Además, se realizaron gráficos de barra o de pastel de manera univariada o multivariada, según se requería, para lograr el análisis que permitiera describir la respuesta de múltiples factores.

\section{ANÁLISIS Y DISCUSIÓN DE LOS RESULTADOS}

\section{Análisis de nivel de satisfacción de implementación de los cursos Moodle}

Como resultado de la encuesta realizada a los estudiantes que cursan las titulaciones que oferta la FAREM-Carazo, se obtuvo los siguientes resultados: El análisis de la variable Valoración Media de la satisfacción de los estudiantes con respecto al uso de la plataforma Moodle durante las clases evidenció que existe un alto nivel de satisfacción entre los estudiantes, con un 75.72\% de tendencia positiva. Esto permite inferir que, los estudiantes se encuentran satisfechos respecto al uso de Moodle como soporte de sus clases presenciales.

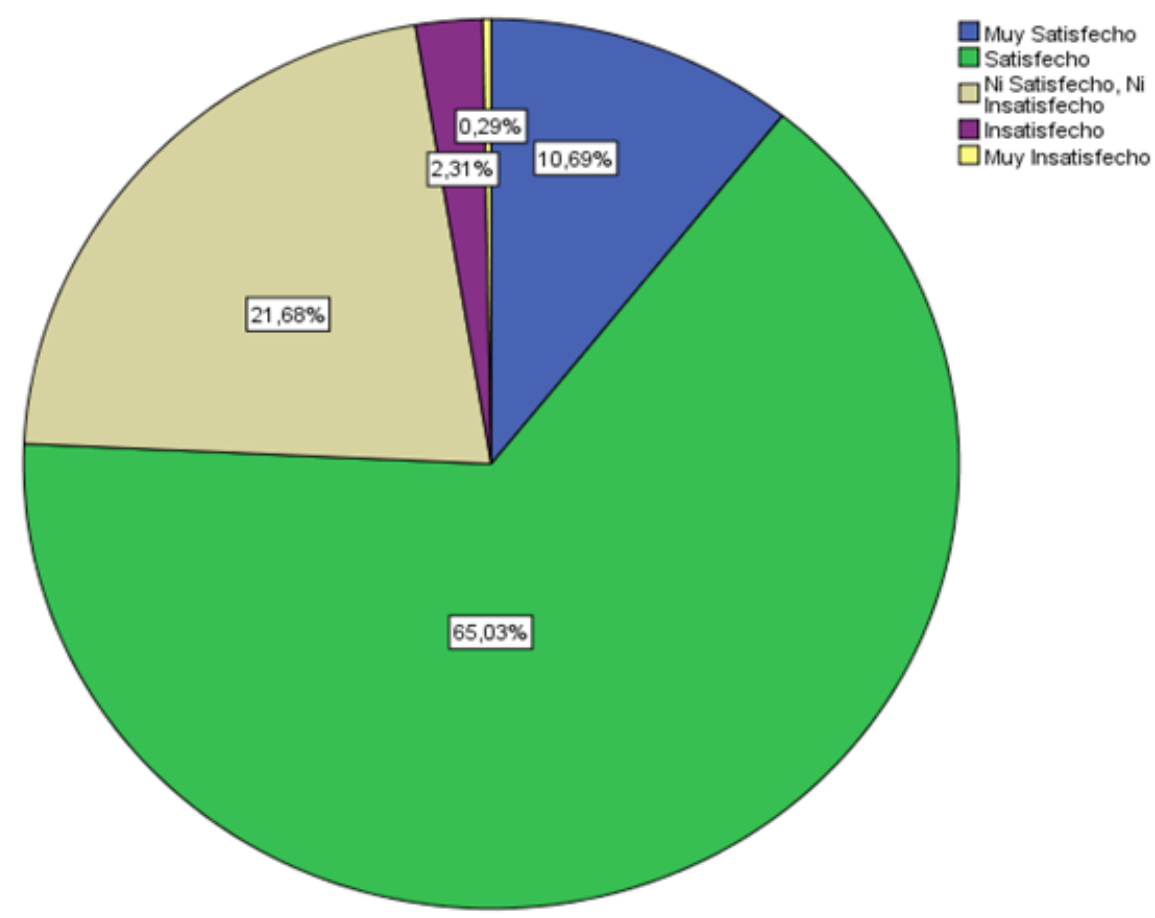

Figura 1: Evaluación de la satisfacción de los estudiantes 


\section{Beneficios y dificultades en el uso de la plataforma MOODLE de parte de docentes de la FAREM-Carazo}

Con respecto a los beneficios y dificultades el maestro Marcos Soza (2017, grupo focal minuto 00:10:36 - 00:10:51) señala lo siguiente: "Moodle es una plataforma que provee más beneficios que dificultades, entre estos beneficios se puede destacar un cambio de paradigma en la metodología, que facilita el proceso de enseñanza-aprendizaje, esto debido a la gran cantidad de herramientas que mejoran aspectos como la retroalimentación, la entrega de tareas y el fomento de actividades colaborativas a distancia entre los docentes y estudiantes, así mismo, le permite al docente proveer una mayor cantidad de recursos didácticos relacionados con la asignatura, por medio de la plataforma, por tanto, ese cambio de paradigma hace que el estudiante se interese más y esté más inmerso en el proceso de enseñanza".

Esta idea es complementada con la percepción general que tienen los estudiantes con respecto a la calidad de procesos de retroalimentación brindada por los docentes, cuyos resultados estadísticos mostraron que existe un alto índice de satisfacción positiva por parte del alumnado.

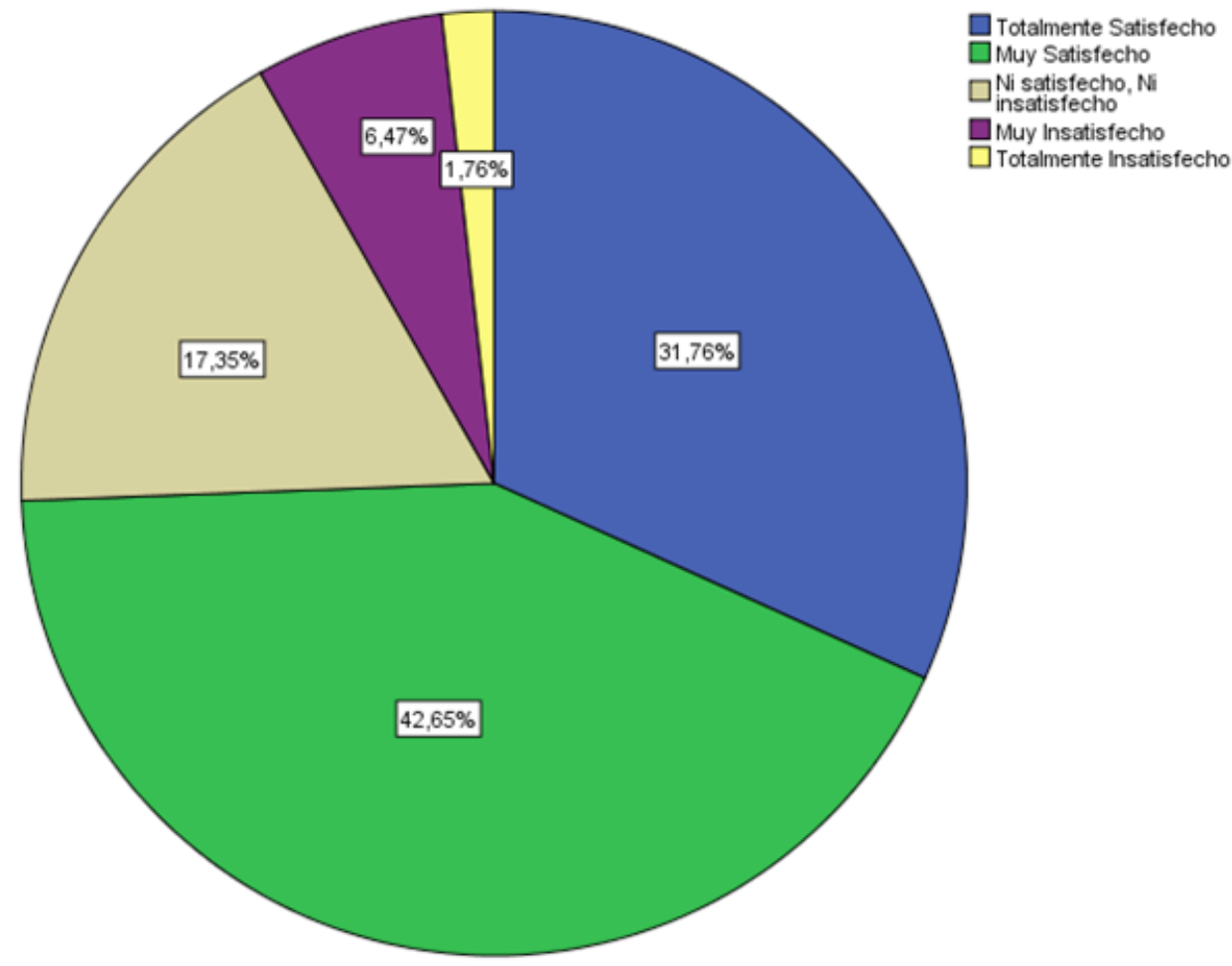

Figura 2: Calidad de la retroalimentación brindada por los docentes

Así mismo, con respecto a la diversidad de recursos didácticos que los docentes suben a la plataforma para ser descargados y de esta manera fortalecer las temáticas impartidas en clases, los resultados muestran nuevamente una tendencia positiva. 


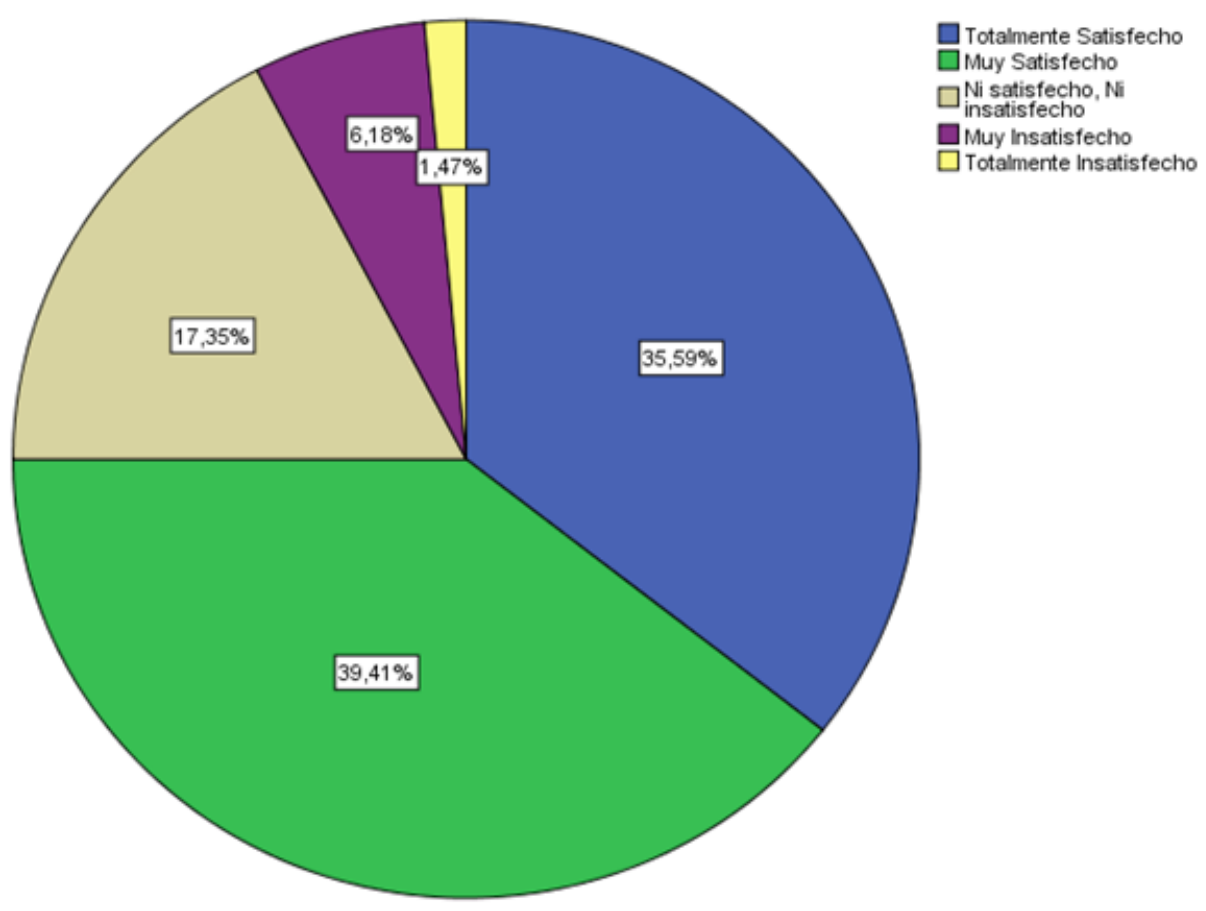

Figura 3: Diversidad de recursos didácticos subidos a la plataforma Moodle por parte de los docentes.

En esta misma línea, el maestro Raúl Medrano (2017, grupo focal minuto 00:12:07 00:12:36) expresó: "Considero que predominan principalmente los aspectos positivos en el uso de la plataforma, estamos definitivamente en la era digital, cada vez se está promoviendo más la educación en línea, es algo que se ha venido mencionando desde el 2007 y ahora más que nunca lo estamos viendo con el concepto de la universidad en línea que ha venido desarrollando el Gobierno Central de Nicaragua".

El maestro Darío Rodríguez (2017, grupo focal minuto 00:14:30 a 00:15:22) comentó: "Una de las partes positivas que hay que destacar, es la comunicación fuera de la clase que tenemos con los estudiantes", así mismo agrega que, otra ventaja que Moodle ofrece es la posibilidad de crear comunidades de aprendizaje dentro del mismo entorno, ya que este permite hacer foros de discusión, comunicación por medio de chats o la realización de tutorías a distancia. Al comparar con los resultados mostrados por la encuesta aplicada a los estudiantes, se tiene que en ambos aspectos, tanto en el uso de chats y foros, existe una tendencia positiva. 


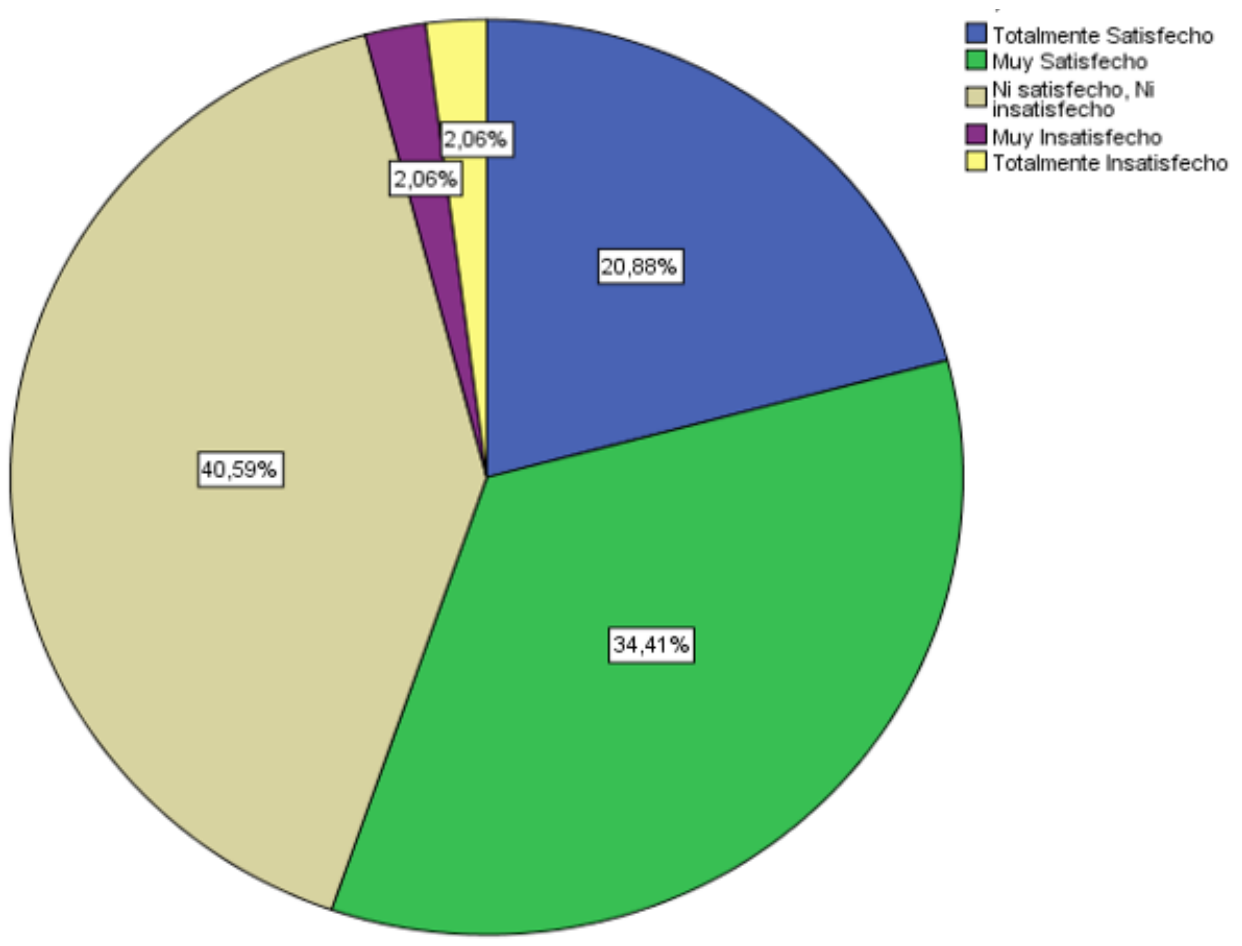

Figura 4: Efectividad de las herramientas de comunicación sincrónica, como los chat.

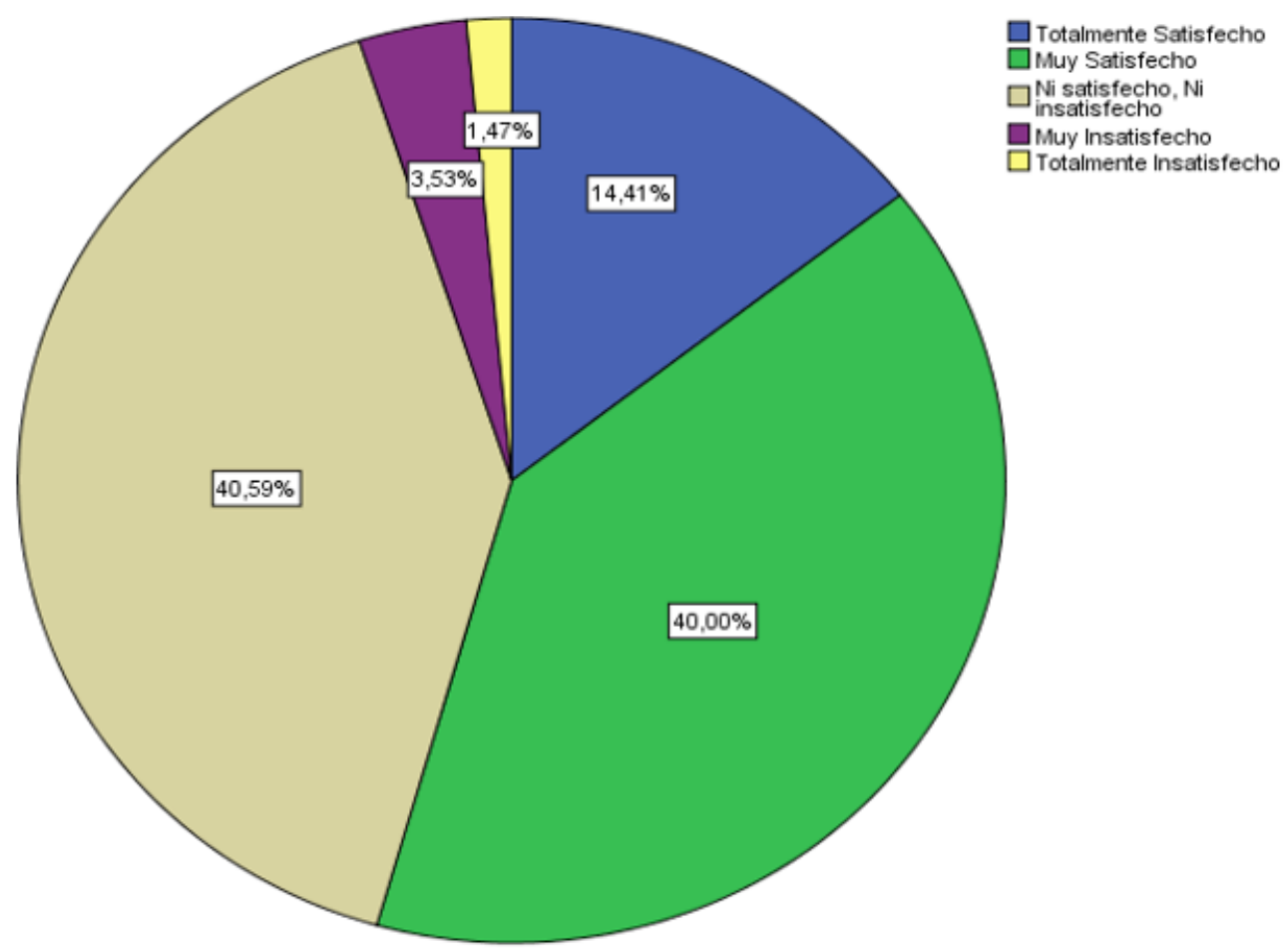

Figura 5: Efectividad de las herramientas de comunicación asincrónica, como los foros.

Por otro lado, el licenciado Roberto Robleto (2017, grupo focal minuto 00:08:46 00:10:04) agregó: "Aunque en primera instancia da la impresión de que el Moodle incurre en una sobrecarga de trabajo para los docentes, este en realidad representa un importante banco 
de datos que facilita la organización y reutilización de la documentación utilizada para las asignaturas, gracias al sistema de subida y organización de archivos que este posee. Adicional a eso, el sistema de manejo de evaluaciones mejora de manera significativa el control de entregas y la gestión de notas, facilitando el trabajo realizado por los docentes".

Con lo que, por lo antes expuesto, se infiere que los beneficios que provee Moodle en los procesos de enseñanza-aprendizaje tienen un alto grado de incidencia positiva, tanto en los docentes como en los estudiantes que se han vistos inmersos en el uso de esta plataforma. Con respecto a las dificultades, se encontró que una de las más frecuentes con respecto al uso de Moodle es la falta de capacitación sobre diseño y gestión de estos cursos.

El maestro Marcos Soza (2017, grupo focal minuto 00:40:50 - 00:41:10) dijo: "Se han recibido capacitaciones, pero la mayoría siempre han sido desde el punto de vista técnico, pero no ha habido capacitaciones que asesoren metodológicamente sobre el uso de la plataforma". Como comenta el maestro Rodríguez, este aspecto es de suma importancia debido a que se pueden incurrir en errores con la estructura del curso y la calidad didáctica de los documentos que se comparten por medio de esta plataforma (Rodríguez, 2017, grupo focal minuto 00:45:10 - 00:41:10).

De la información arrojada por los instrumentos aplicados, se observa cómo los participantes concuerdan en que Moodle es una herramienta de suma importancia para mejorar los procesos educativos, ya que esta plataforma provee una gran cantidad de beneficios que se pueden explotar para mejorar la experiencia de los estudiantes en clases y de esta manera, hacer frente a algunas de las demandas de la actual sociedad del conocimiento y de la información. Esto coincide con el planteamiento de Rodríguez, Migueláñez y Sánchez (2014), quienes explican que Moodle pone su mayor énfasis en la idea de interacción con los demás, mediante comunidades de aprendizaje que favorecen el trabajo colaborativo que se traduce, finalmente, en un aprendizaje significativo, gracias al intercambio y conexión de conocimientos que favorecen y facilitan su construcción a partir de los previamente adquiridos.

Entre las dificultades que los docentes expresan sobre el manejo, diseño y gestión de la plataforma Moodle, las más predominantes son: las fallas técnicas referentes a la accesibilidad y conexión de Internet, así mismo, la falta de capacitación al docente y la resistencia al cambio por parte de un sector del profesorado que se aferra a los métodos de enseñanza tradicional. Esto tiene concordancia con lo plasmado por Cabero (2011), en cuya obra están plasmadas estas y otras dificultades expuestas por los docentes de la FAREM-Carazo. 


\section{Características comunes en los cursos que desarrollan los docentes de la FAREM- Carazo en la Plataforma Moodle}

Con respecto a las características que poseen los cursos Moodle implementados por los docentes de la FAREM-Carazo, al realizar una revisión de un segmento de estos, se obtuvieron los siguientes resultados:

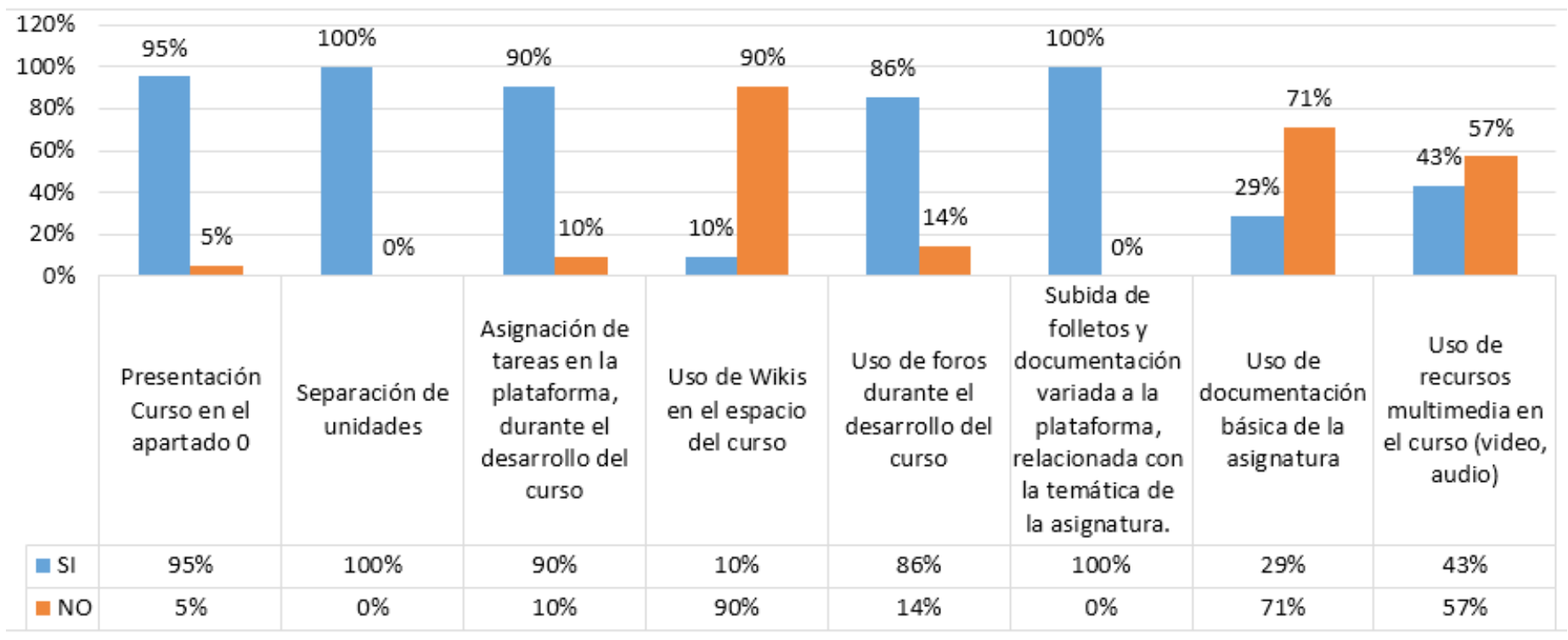

Figura 6: Características de los cursos Moodle implementados en la FAREM-Carazo, en el primer semestre del 2017.

$\mathrm{Al}$ analizar las características, se observa que, a pesar de que no existe una correcta orientación metodológica con respecto a cómo se debe crear y diseñar un curso Moodle, los docentes de la FAREM-Carazo han asumido tendencias comunes en la forma en que se crean los cursos, los cuales poseen rasgos compartidos que le permiten al estudiante adecuarse rápidamente al entorno. Pero a pesar de estas similitudes, los hallazgos encontrados muestran un bajo nivel de interacción por parte de los estudiantes, por ello es necesario que los docentes no solo sean administradores de los cursos que implementan en la plataforma, sino que adicional a esto, sean un agente motivador, que guíe a los estudiantes a tener una mejor experiencia en el uso de la plataforma y de esta manera, incidir de manera significativa durante su formación.

\section{CONCLUSIONES}

Después de haber analizado los resultados de los instrumentos aplicados tanto a docentes como estudiantes de la UNAN-Managua, FAREM-Carazo, se logró determinar que el nivel de aceptación tanto de los docentes, como de los estudiantes con respecto al uso de la plataforma Moodle como soporte a las clases presenciales es bastante alto, y en su mayoría, están altamente satisfechos con los beneficios y herramientas que esta ofrece.

Así mismo, se logró determinar que Moodle es una plataforma que ofrece una gran cantidad de beneficios y que su utilización como herramienta de soporte a las clases presenciales 
permite mejorar los procesos de interacción, comunicación e información. Pero a pesar de que es una herramienta que ofrece grandes ventajas, durante todo el tiempo que esta ha estado en línea dentro de la facultad, se han hecho presentes muchas dificultades; por un lado, las que son de carácter técnico, que tienen que ver con cuestiones de conectividad y accesibilidad a la plataforma, y por otro lado, las que son de carácter metodológico, las que tienen que ver con limitaciones de los docentes con respecto al diseño y gestión de los cursos, esto debido a la falta de una normativa que permita estandarizar y guiarlos acerca de la forma en que un curso debe ser implementado en Moodle.

Con la información recopilada y la revisión de las características de los cursos Moodle implementados por los docentes de la UNAN-Managua, FAREM-Carazo, se concluye que, a pesar de la falta de una correcta orientación metodológica acerca del diseño y gestión de cursos Moodle, se logra identificar que existen características metodológicas comunes entre los cursos creados por los docentes, entre las que destacan el formato del curso, la inclusión de tareas y foros de discusión, los cuales dan la posibilidad de incrementar el nivel de interacción de los estudiantes en las actividades del curso.

Al realizar un análisis detenido del nivel de interacción de los estudiantes con las asignaciones implementadas en cada uno de los cursos, se encontró que a pesar del alto nivel de aceptación que tienen los estudiantes en relación al uso de la plataforma, estos no interactúan frecuentemente con esta y en muchos de los casos, no cumplen con las actividades propuestas por el docente.

A partir de los hallazgos del estudio, se genera una propuesta de normativa para el diseño y gestión de cursos Moodle en la FAREM-Carazo, UNAN-Managua, que contempla aspectos técnicos, metodológicos y de procedimiento, relacionados con el quehacer de los diversos actores implicados en el uso de esta importante herramienta tecnológica: Personal de soporte, docentes y estudiantes.

\section{REFERENCIAS}

Cabero, J. (2006). Bases Pedagógicas Del E-Learning. Revista Universitaria $y$ Sociedad del Conocimiento Vol. 3,10.

Fernández, S. (2006). Herramientas Digitales de Comunicación para la Educación Superior.

Galindo, M. (1996). Métodos y Técnicas de Investigación. Trillas.

Gómez-López, J. R., Reyes-Lizárraga, J. O. \& Tirado-Osuna, I. (2015). Análisis de Uso de la Plataforma Moodle en Estudiantes Universitarios.

Hernández, R., Fernández, C. \& Baptista, C. (2014). Metodología de la Investigación. México: Mac Graw Hill.

Rodríguez, A. I., Migueláñez, S. O., Sánchez, E. M. \& Marcos, J. J. (2014). Evaluar para Optimizar el Uso de la Plataforma Moodle. Salamanca: Universidad de Salamanca. 